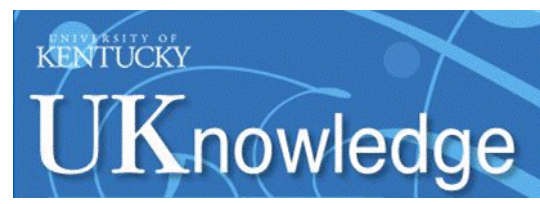

University of Kentucky

UKnowledge

\title{
Life-Shortening Wolbachia Infection Reduces Population Growth of Aedes aegypti
}

\author{
Eunho Suh \\ University of Kentucky, eunho.suh@uky.edu \\ David R. Mercer \\ University of Kentucky \\ Stephen L. Dobson \\ University of Kentucky, sdobson@uky.edu
}

Follow this and additional works at: https://uknowledge.uky.edu/entomology_facpub

Part of the Bacteria Commons, Entomology Commons, and the Population Biology Commons

Right click to open a feedback form in a new tab to let us know how this document benefits you.

\section{Repository Citation}

Suh, Eunho; Mercer, David R.; and Dobson, Stephen L., "Life-Shortening Wolbachia Infection Reduces Population Growth of Aedes aegypti" (2017). Entomology Faculty Publications. 179.

https://uknowledge.uky.edu/entomology_facpub/179

This Article is brought to you for free and open access by the Entomology at UKnowledge. It has been accepted for inclusion in Entomology Faculty Publications by an authorized administrator of UKnowledge. For more information, please contact UKnowledge@lsv.uky.edu. 


\section{Life-Shortening Wolbachia Infection Reduces Population Growth of Aedes aegypti}

\section{Digital Object Identifier (DOI)}

https://doi.org/10.1016/j.actatropica.2017.05.015

\section{Notes/Citation Information}

Published in Acta Tropica, v. 172, p. 232-239.

(C) 2017 Elsevier B.V. All rights reserved.

This manuscript version is made available under the CC-BY-NC-ND 4.0 license http://creativecommons.org/licens es/by-nc-nd/4.0/.

The document available for download is the author's post-peer-review final draft of the article. 
Published in final edited form as:

Acta Trop. 2017 August ; 172: 232-239. doi:10.1016/j.actatropica.2017.05.015.

\title{
Life-shortening Wolbachia infection reduces population growth of Aedes aegypti
}

\author{
Eunho Suh ${ }^{\mathrm{a}, 1,{ }^{*}, \text { David R. Mercer }}{ }^{\mathrm{a}, 2}$, and Stephen L. Dobson ${ }^{\mathrm{a}}$ \\ aDepartment of Entomology, University of Kentucky, Lexington, KY 40546 \\ ${ }^{1}$ Department of Entomology, Center for Infectious Disease Dynamics, 001 Merkle Lab, The \\ Pennsylvania State University, University Park, PA 16802
}

\begin{abstract}
Wolbachia bacteria are being introduced into natural populations of vector mosquitoes, with the goal of reducing the transmission of human diseases such as zika and dengue fever. The successful establishment of Wolbachia infection is largely dependent on the effects of Wolbachia infection to host fitness, but the effects of Wolbachia infection on the individual life history traits of immature mosquitoes can vary. Here, the effects of life-shortening Wolbachia (wMelPop) on population growth of infected individuals were evaluated by measuring larval survival, developmental time and adult size of Aedes aegypti in intra- (infected or uninfected only) and inter-group (mixed with infected and uninfected) larval competition assays. At low larval density conditions, the population growth of $w$ MelPop infected and uninfected individuals was similar. At high larval densities, wMelPop infected individuals had a significantly reduced population growth rate relative to uninfected individuals, regardless of competition type. We discuss the results in relation to the invasion of the wMelPop Wolbachia infection into naturally uninfected populations.
\end{abstract}

\section{Graphical abstract}

Wolbachia infection reduces mosquito population growth at high larval density conditions

\footnotetext{
"Corresponding author: Department of Entomology, Center for Infectious Disease Dynamics, The Pennsylvania State University, 001 Merkle Lab, University Park, PA 16802, Telephone: 814-867-4191, eus57@psu.edu.

${ }^{2}$ Deceased

Publisher's Disclaimer: This is a PDF file of an unedited manuscript that has been accepted for publication. As a service to our customers we are providing this early version of the manuscript. The manuscript will undergo copyediting, typesetting, and review of the resulting proof before it is published in its final citable form. Please note that during the production process errors may be discovered which could affect the content, and all legal disclaimers that apply to the journal pertain.

Competing interests

The authors declare that they have no competing interests. SLD is a Full Professor at the University of Kentucky and a paid employee of MosquitoMate, a for-profit company. This does not alter our adherence to all Acta Tropica policies.

Authors' contribution

ES, DRM and SLD conceived and designed the study. ES performed the experiments. ES and SLD analyzed the data. ES and SLD wrote the paper.
} 

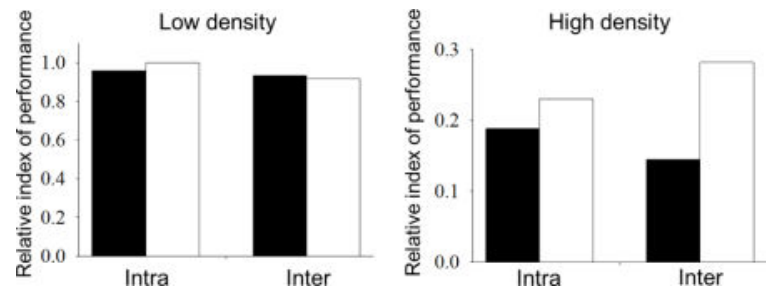

\section{Keywords}

population replacement; dengue; larval competition; fitness cost; population dynamics; population growth

\section{Introduction}

Wolbachia are maternally inherited intracellular bacteria that occur naturally in a wide range of invertebrate species (Hilgenboecker et al., 2008; Zug and Hammerstein, 2012). Wolbachia infections induce diverse reproductive effects in hosts, such as feminization, parthenogenesis, male killing, and cytoplasmic incompatibility (CI) (Jeong and Suh, 2008; Werren et al., 2008). In mosquitoes, CI causes a reduction of progeny from uninfected females that mate with infected males, which can promote the invasion of Wolbachia infected hosts into an uninfected population through the relative reproductive advantage conferred by the Wolbachia infection (McGraw and O'Neill, 2013).

Wolbachia infections can lower the ability of mosquitoes to transmit infections of important human pathogens, such as zika, dengue, chikungunya, yellow fever virus and malaria (Aliota et al., 2016; Bian et al., 2013; Bian et al., 2010; Blagrove et al., 2012; Dutra et al., 2016; Hedges et al., 2008; Kambris et al., 2010; Kambris et al., 2009; Moreira et al., 2009; Van den Hurk et al., 2012; Walker et al., 2011; Ye et al., 2015). Accordingly, Wolbachia infection has been proposed for an applied use and utilized as a bio-control agent to reduce human disease transmission (Bourtzis et al., 2014; Iturbe-Ormaetxe et al., 2011). In two Australian cities, artificial Wolbachia infections using a disease blocking Wolbachia strain (wMel) were successfully established in naturally uninfected field populations of Aedes aegypti (Hoffmann et al., 2014b; Hoffmann et al., 2011; Walker et al., 2011). The goal of establishing Wolbachia in the A. aegypti population was to reduce potential for dengue virus transmission, because Wolbachia-infected females have been shown to exhibit lower infection rates of the virus (Frentiu et al., 2014; Hoffmann et al., 2014b; Hoffmann et al., 2011; Walker et al., 2011; Ye et al., 2015).

wMelPop Wolbachia strain holds a great potential for controlling vector borne diseases including dengue as the infection not only reduces the number of disease carrying mosquitoes due to the life-shortening phenotype in adult mosquitoes (McMeniman et al., 2009), but also exhibits stronger blocking activity against viral infections than similar Wolbachia strains, including wMel (Chrostek et al., 2013; Ferguson et al., 2015; Ye et al., 2013). In addition to the proposed use of $w$ MelPop infection in population replacement strategies, feasibilities for a novel suppression strategy are being investigated. The suppression strategy targets areas with distinct wet/dry seasons and limited migration of 
mosquitoes where an establishment of $w$ MelPop infection during wet seasons can potentially lead to a reduction or extinction of local vector population during dry seasons as the infection causes egg mortality (McMeniman and O'Neill, 2010; Rasic et al., 2014; Ritchie et al., 2015; Yeap et al., 2011). However, the wMelPop strain also imposes diverse fitness costs on hosts in multiple life history including longevity in adult and egg stage (McMeniman et al., 2009; McMeniman and O’Neill, 2010; Yeap et al., 2011), larval survival from competitive interactions and larval behavior (Suh and Dobson, 2013), larval tolerance to starvation (Ross et al., 2016), larval development time (McMeniman and O'Neill, 2010; Ross et al., 2014; Yeap et al., 2011), blood feeding success (Turley et al., 2009), clutch size and/or egg hatch rate (McMeniman et al., 2011; Suh et al., 2016; Suh et al., 2009), or female size and oviposition success (Ross et al., 2014; Yeap et al., 2014), which can all hinder Wolbachia invasion and establishment in the targeted mosquito populations (Nguyen et al., 2015).

The competitiveness of immature mosquitoes often plays a crucial role in determining the geographical distribution of mosquitoes (Braks et al., 2004; Juliano, 1998; Livdahl and Willey, 1991). Relatedly, studies that have previously characterized the effects of Wolbachia on the fitness of immature insects highlight the importance of immature competitiveness on the invasion dynamics of Wolbachia (Crain et al., 2011; Gavotte et al., 2010; Gavotte et al., 2009; Hancock et al., 2016; Ross et al., 2016; Ross et al., 2014; Suh and Dobson, 2013). The primary traits associated with the development of immature mosquitoes include survival rates, developmental time, and adult size. These traits are key components in determining population growth rate, which measures changes in population size over time, providing crucial information in understanding population dynamics (Livdahl and Sugihara, 1984). The effects of Wolbachia on individual life history traits can range from negative to positive (Gavotte et al., 2010; Gavotte et al., 2009; McMeniman and O'Neill, 2010; Ross et al., 2016; Ross et al., 2014; Suh and Dobson, 2013; Yeap et al., 2011), suggesting an estimation of "net impact" on infected populations can be helpful for understanding the population dynamics.

Here, we examined the effects of wMelPop infection, a variant strain of the wMel Wolbachia type (McMeniman et al., 2008; Min and Benzer, 1997), on the traits that are predicted to affect the relative fitness of immature $A$. aegypti, the primary dengue vector mosquito. We assessed the impact of $w$ MelPop infection on intra- (infected or uninfected only) and intergroup (mixed with infected and uninfected) competition under two larval density conditions by examining immature survival, developmental time, and adult size. These parameters were then used to estimate the relative population growth rate of infected individuals. We show that population growth is not affected by infection status at low larval density, while the population growth of an infected population is significantly reduced at high larval density, regardless of the competition type. We discuss the results in relation to applied strategies intended to improve public health outcomes, to optimizing approaches for establishing infections, and to the population dynamics following the establishment of the infection. 


\section{Materials and methods}

\subsection{Insect strains}

Experiments used $A$. aegypti colonies PGYP1, which was infected with the wMelPop strain, and PGYP1.tet, which was the PGYP1 cured of Wolbachia by tetracycline treatment (McMeniman et al., 2009). All maintenance and experiments were conducted at $28 \pm 2{ }^{\circ} \mathrm{C}$, $75 \pm 10 \% \mathrm{RH}$, and a photoperiod of 18:6 h (L:D) as described previously (McMeniman et al., 2009). In brief, larvae were fed fish food (TetraMin Tropical Tablets, Tetra, Germany) in $400 \mathrm{ml}$ DI (deionized) water ad libitum. Adults were maintained in $30 \times 30 \times 30 \mathrm{~cm}$ cages with constant access to a $10 \%$ sucrose solution. Human blood (Blood center, Lexington, KY) was provided using Hemotek artificial blood feeding system (Discovery Workshops, Accrington, UK) for blood feeding.

\subsection{Larval competition assays and estimation of wing size, developmental time, and survival}

To determine the optimal rearing conditions for immature $A$. aegypti, 300 larvae were reared using a series of different resource regimes. Fifty PGYP1 larvae (within $2 \mathrm{~h}$ of hatching) were transferred into containers (Mosquito Breeders; BioQuip, Rancho Dominguez, CA) with $200 \mathrm{ml}$ DI water, and were provided with 10, 40, 70, 100, 130 or $160 \mathrm{mg}$ fish food every third day until pupation. Eclosing adults were counted, and their sex and developmental time (i.e., time to emergence) were recorded. The food level defined as the optimal amount of food required for 50 infected larvae with higher survival and shorter developmental time was determined as $70 \mathrm{mg}$ (Figure $\mathrm{S} 1$ ). We then used this amount of food when examining the effect of $w$ MelPop infection on the development of $A$. aegypti larvae under varying competition conditions.

For larval competition assays, defined numbers of PGYP1 and PGYP1.tet larvae (within $2 \mathrm{~h}$ of hatching) were placed in containers with $200 \mathrm{ml}$ DI water and $70 \mathrm{mg}$ fish food, given every third day as described above. Two larval density conditions (total numbers for low $=$ 50 larvae, high $=400$ larvae) were compared following similar conditions that were described in a previous study (Gavotte et al., 2010). Each density included three treatments for larval competition: PGYP1 only, PGYP1.tet only (i.e., intra-group competition) and 1:1 ratio of PGYP1+PGYP.tet (i.e., inter-group competition). Each of the six treatments was replicated four times. Eclosing adults were collected daily until no viable immature individuals remained. The sex, eclosion time, and wing size of emerged adults were recorded.

The wing size and Wolbachia infection status were determined for a subset of eclosed adults, which were selected by a sampling approach that was designed to minimize sampling bias. Females and males were each divided into five equally sized groups, according to eclosion time. For intra-group competition at low larval density (LD) condition, five females and five males were randomly selected from each of the resulting groups. To collect a similar sample number for each mosquito strain (i.e., PGYP1 and PGYP1.tet) from the inter-group competition, the sample size was doubled (i.e., ten females and ten males from each group). For the high larval density (HD) condition, a similar sampling approach was used, but the 
sample size was increased by four times for each treatment (i.e., 40 and 80 samples from intra- and inter-group competitions, respectively).

To measure wing size, images of wings (right side) were captured using a zPix MM-640 microscope (Carson Optical, Hauppauge, NY), and the wing length (alula to wing tip) was estimated using ImageJ software (Barboriak et al. 2005).

For PCR, DNA was extracted from sampled adult mosquitoes as described previously (Brelsfoard et al., 2008). PCR amplification was performed in $25 \mu \mathrm{l}$ reaction volumes using Taq DNA polymerase (Invitrogen Life Technologies, Carlsbad, CA) with 16s Wolbachia specific (Werren and Windsor, 2000) and CO1 universal primers (Hebert et al. 2003), to determine both Wolbachia infection and template quality in a mixture of $17.5 \mu \mathrm{l} \mathrm{H} \mathrm{H}_{2} \mathrm{O}, 2.5 \mu \mathrm{l}$ $10 \times$ buffer, $0.8 \mu \mathrm{l}$ of dNTP $(10 \mathrm{mM}), 0.5 \mu \mathrm{l}$-specf, W-specr, CO1f and CO1r primers (10 $\mu \mathrm{M}$ each), $0.2 \mu \mathrm{l}$ Taq and $2 \mu \mathrm{l}$ of DNA template. A MJ Research PTC-200 Thermal Cycler (Bio-Rad Laboratories, Hercules, CA) was used to perform PCR reactions with $94^{\circ} \mathrm{C}$ for 2 min and 38 cycles of $94^{\circ} \mathrm{C}$ for $2 \mathrm{~min}, 55^{\circ} \mathrm{C}$ for $45 \mathrm{sec}$ and $72^{\circ} \mathrm{C}$ for $1.5 \mathrm{~min}$, followed by $72{ }^{\circ} \mathrm{C}$ for $10 \mathrm{~min}$.

Mean developmental time was estimated based on the eclosion time of all collected individuals for intra-group competitions. For inter-group competitions, mean developmental time was estimated from the subsampled individuals (as described above) with known infection status and eclosion time.

To calculate the survival of PGYP1 and PGYP1.tet individuals in an intra-group competition, the number of collected adults was divided by the initial number of larvae used in the experiments (i.e., 50 for LD and 400 for HD condition). To estimate the relative survival of PGYP1 and PGYP1.tet individuals from the inter-group competition, the following equations were used to estimate the ratio of PGYP1: PGYP1.tet individuals for each sex. $E_{f_{P G Y P 1}}$ is the estimated proportion of PGYP1 females (i.e., the proportion of PGYP1 females multiplied by the sex ratio), expressed as:

$$
E_{f_{P G Y P 1}}=\left(\frac{f_{P G Y P 1}}{f_{P G Y P 1}+f_{P G Y P 1 . t e t}}\right)\left(\frac{f}{f+m}\right) \quad \text { Equation 1 }
$$

where $f_{P G Y P 1}$ and $f_{P G Y P 1 . t e t}$ are the number of PGYP1 and PGYP1.tet females, respectively, as determined from the PCR test of the sampled females. $f$ and $m$ are the total number of eclosing females and males, respectively. A similar method was used to estimate the proportion of PGYP1.tet females $\left(E_{f_{P G Y P 1 . t e t}}\right)$, PGYP1 males $\left(E_{m_{P G Y P 1}}\right)$ and PGYP1.tet males $\left(E_{m_{P G Y P 1 . t e t}}\right)$.

The survival of PGYP1 or PGYP1.tet individuals from inter-group competition was calculated using Equation 2. $E_{P G Y P 1}$ is the estimated total number of eclosed, PGYP1 individuals expressed as: 


$$
E_{P G Y P 1}=\left(E_{f_{P G Y P 1}}+E_{m_{P G Y P 1}}\right)(f+m) \quad \text { Equation } 2
$$

Thus, survival of PGYP1 individuals was calculated by dividing $E_{P G Y P 1}$ by the initial larval number (i.e., 25 for LD and 200 for HD condition). A similar method was used to estimate the total number of eclosed PGYP1.tet individuals $\left(E_{P G Y P 1 . t e t}\right)$ and calculate the survival of PGYP1.tet individuals from the initial number.

To better understand sex specific survival of PGYP1 and PGYP1.tet individuals from intergroup competition, departure from equal emergence was calculated.

Assuming an equal sex ratio and survival of PGYP1and PGYP1.tet individuals, the proportion of each would be: $E_{f_{P G Y P 1}}=E_{f_{P G Y P 1 . t e t}}=E_{m_{P G Y P 1}}=E_{m_{P G Y P 1 . t e t}}=0.25$. Thus, the departure from equal emergence for PGYP1 females $\left(D_{f_{P G Y P 1 . t e t}}\right)$ was calculated as $4 E_{f_{P G Y P 1}}-1$, with similar calculations made for PGYP1.tet females $\left(D_{f_{P G Y P 1 . t e t}}\right)$, PGYP1 males $\left(D_{m_{P G Y P 1 . t e t}}\right)$ and PGYP1.tet males $\left(D_{m_{P G Y P 1 . t e t}}\right)$.

\subsection{Population growth rate}

To examine the combined impact of $w$ MelPop infection on survival, developmental time, and expected fecundity of adult females using a size parameter, a simplified index of performance (I) was calculated for PGYP1 and PGYP1.tet individuals in intra- and intergroup competitions to simulate the population growth rate as described in a previous study (Koenraadt et al., 2010).

$$
I=\frac{\ln \frac{1}{N_{0}} \sum_{x} A_{x} \bar{w}_{x}}{\sum_{x} x A_{x} \bar{w}_{x} / \sum_{x} A_{x} \bar{w}_{x}} \quad \text { Equation } 3
$$

where $N_{O}$ is the initial number of females (assumed to be $50 \%$ of the initial immature number in the experiments), $A_{X}$ is the number of adult females produced at time $X$, and $\bar{w}$ is the mean size of the emerging females.

\subsection{Statistical analyses}

The major objective for statistical analyses was to examine how Wolbachia infection status and competition type (i.e., intra- and inter-group competition) affected survival of immature individuals, developmental time, adult size, and population growth at two different larval densities, and similar statistical methods were applied as previously described (Koenraadt et al., 2010). Survival data were analyzed using a generalized linear model (GLM, binomial distribution with Logit link; JMP 8.0.1; SAS Institute, Cary, NC) to test the effect of infection status and competition type for each larval density. Multiple post-hoc contrast tests were conducted when a significant interaction effect was observed between infection status and competition type with Bonferroni correction. Specifically, in order to characterize relative survival in an inter-group competition, ANOVA (JMP 8.0.1; SAS Institute, Cary, 
NC) was used to examine the effect of infection status and competition type after square root arcsine transformation of survival data for each larval density. Similarly, the effect of infection status and competition type on developmental time, adult size, and performance index was examined using ANOVA (JMP 8.0.1; SAS Institute, Cary, NC) for each sex or larval density as needed. In particular, performance index values were log transformed to meet the assumption of ANOVA (e.g., normality, equal variance, etc.). Subsequently, posthoc Tukey-Kramer Honestly Significant Difference (HSD) test was performed if a significant interaction effect was observed between infection status and competition type.

\section{Results}

\subsection{Survival}

Survival of PGYP1 and PGYP1.tet individuals from intra- and inter-group competition was compared to examine for an effect of infection status and competition type (i.e., intra- and inter-group competition) under two larval density conditions. Immature mosquito survival under low density (LD) conditions was significantly higher than that observed at high density (HD) conditions (LR- $\chi^{2}=30.56, p<0.0001$ ), and survival ranged from $87 \%$ to $96 \%$ with LD conditions (Figure 1A), and from 75\% to 93\% with HD conditions (Figure 1B). Generalized linear model analyses revealed that under LD conditions, PGYP1 individuals had lower survival rates regardless of competition type (Table 1). Under HD conditions, a significant interaction effect was observed between infection status and competition type (Table 1). Pairwise post-hoc contrast tests showed that PGYP1.tet individuals from intergroup competitions had significantly higher survival rates than the other three groups ( $p<$ 0.0001; Bonferroni corrected) (Figure 1B). An ANOVA test examining the effect of infection status and sex on the relative survival in an inter-group competition showed that PGYP1.tet relative to PGYP1 individuals, and males relative to females showed higher survival rates under HD conditions, while no such effect was observed with LD conditions (Table 2). These results were represented as departure data (assuming a 1:1 ratio between PGYP1 and PGYP1.tet, or females and males, Figure 1C).

\subsection{Developmental time}

Due to a significant interaction between larval density conditions and one of the main variables of interest (i.e., infection status and competition type), analyses of developmental time were conducted separately for each density condition. Overall, the variation in development time of PGYP1 individuals was increased relative to PGYP1.tet as more PGYP1 emerged late relative to PGYP1.tet (Figure S2). Under LD conditions, males developed faster than females, and PGYP1.tet individuals developed faster than PGYP1 (Table 3, Figure 2A and 2B). Similar to the LD conditions, under HD conditions males developed faster than females, but a significant interaction was observed between infection status and competition type (Table 3, Figure 2C and 2D). A post-hoc Tukey-Kramer HSD test showed that, relative to PGYP1 individuals, PGYP1.tet developed faster in an intragroup competition. Meanwhile, inter-group competition increased the difference in developmental time between the PGYP1 and PGYP1.tet individuals independently of sex (Figure 2C and 2D). 


\subsection{Adult size}

Because of significant interactions between sex or larval density and main variables of interest, analyses of adult size (wing length) were conducted separately for each sex and larval density condition. Because significant interactions between infection status and competition type were observed for both females and males with LD and HD conditions (Table 4), post-hoc Tukey-Kramer HSD tests were conducted for all four cases. Under LD conditions, PGYP1 and PGYP1.tet females had similar sizes in intra-group competitions, but the size difference was significant in an inter-group competition (Figure 3A). A similar pattern was observed for males, but the size of PGYP1 and PGYP1.tet males did not differ significantly in an inter-group competition (Figure 3B). In contrast to LD conditions, PGYP1 individuals achieved a size advantage in inter-group competitions under HD conditions when compared to intra-group competitions. Specifically, the size of PGYP1 and PGYP1.tet females was similar in an inter-group competition, while PGYP1.tet females were larger than PGYP1 females in an intra-group competition (Figure 3C). Similarly, PGYP1 males were larger than the PGYP1.tet males in an inter-group competition, but no size difference was observed in intra-group competitions (Figure 3D).

\subsection{Population growth}

In order to evaluate the overall impact of Wolbachia infection on the reproductive success of immature individuals and consequent population dynamics, the population growth of individual females (i.e., population growth rate) was estimated by calculating the performance index derived from individual traits such as survival and developmental time of immature mosquitoes, and size of adult mosquitoes (Figure 4). Analyses of the performance indices were conducted separately for each larval density condition because of significant interactions between larval density and the main variables of interest. At LD condition, the infection status or competition type had no effect on the performance index, whereas infection status at HD condition had a significant effect, indicating that infected individuals had lower population growth rates regardless of competition type when larval density was high (Table 5). The relative performance index was compared by setting the index value of PGYP1.tet individuals in an intra-group competition under LD condition as the standard index (defined as 1), and then the performance index of other groups was calculated based on the standardized relative index (Figure 4).

\section{Discussion}

Population replacement strategies include releases of Wolbachia infected individuals into the field, where various types of larval competition can occur among infected and/or uninfected wild type individuals. While the infection cost on larval fitness can be a major impediment for Wolbachia invasion (Crain et al., 2011; Hancock et al., 2016), information on the effects of Wolbachia on immature mosquitoes is limited in relation to understanding population dynamics. Here, we examined the effects of $w$ MelPop infection on the relative fitness of immature $A$. aegypti in intra- and inter-group competitions at two larval density conditions, and estimated relative population growth of infected individuals. While examinations on any possibility of genetic drift in the mosquito lines might be useful, the results show that the wMelPop infection on individual life-history traits was mostly detrimental, which is 
consistent with prior studies (McMeniman and O'Neill, 2010; Ross et al., 2014; Suh and Dobson, 2013; Yeap et al., 2011). The cost of wMelPop infection was manifested in general as lower survival and delayed development at LD conditions (Figure 1A, 2A and 2B), but a potential trade-off between these traits was observed at HD conditions. For instance in intragroup competitions, larval competition effects would be mitigated as PGYP1 larvae develop less synchronously (Figure S2B) possibly due to a variation in developmental time associated with Wolbachia infection in individual larvae, while the effects would be intensified as PGYP1.tet larvae develop more synchronously (Figure S2B). Consistent with this hypothesis, in intra-group competitions, reduction in survival from LD to HD conditions was greater in PGYP1.tet (96\% to 79\%; Figure 1A and 1B) relative to PGYP1 (87\% to 79\%; Figure 1A and 1B). Similarly, in inter-group competitions at HD conditions, PGYP1.tet larvae would develop faster (Figure 2C and 2D) by securing more resource as PGYP1 larvae suffer infection cost on behavioral traits (e.g., delayed foraging) (Suh and Dobson, 2013). Consequently, the difference in development time between PGYP1 and PGYP1.tet was accentuated in inter-group competitions (Figure 2C and 2D), and such developmental asynchrony could be associated with overall higher survival in the mixed group relative to PGYP1 or PGYP1.tet only (Figure S2B). However, as constant amount of food is provided per container over time, late developing larvae (e.g., PGYP1) could secure more food per larva relative to early developing larvae (e.g., PGYP1.tet), which may account for the size advantage in PGYP1 individuals from inter-group competitions relative to intra-group competitions at HD conditions (Figure 3C and 3D).

The PGYP1 males with increased size at HD conditions (Figure 3D) resulting from direct competition may facilitate the spread of Wolbachia infection with enhanced male performance by effective sterilization of wild type females (e.g., larger amount of sperm for greater mating capacity and increased mating competitiveness), however the relative contribution of such effects on the Wolbachia invasion dynamics needs further examinations. At LD conditions, the effects of Wolbachia infection on individual traits were less detrimental on the survival of PGYP1 females (Figure 1C) as opposed to the negative effects on developmental time and size (Figure 2A and 3A). As suggested by a previous model study, both fecundity and survival of females have major effects on the population replacement success (Crain et al., 2011). Thus, an understanding of how these individual parameters interact to affect the population dynamics of infected individuals is important. Moreover, an estimation of the population growth rate that reflects all observed parameters would be useful for evaluating the overall impact of Wolbachia infection. Here, we demonstrate that when the competition level is low the relative population growth of PGYP1 individuals is similar to that of PGYP1.tet regardless of competition type (Figure 4A), but it is significantly reduced by $18 \%$ (intra-group competition) to $49 \%$ (inter-group competition) with HD conditions (Figure 4B). Our result suggests that the spread of wMelPop infection in the field could be facilitated when larval competition is suppressed.

The results from intra-group competitions under HD conditions provide insights into the population size and vectorial capacity of infected individuals following establishment of wMelPop infection in field populations. Larval diet level did not affect ability to block dengue virus infection in wMelPop infected $A$. aegypti (Kho et al., 2016), suggesting the blocking ability may not be affected by the reduced size of infected females resulting from 
the increased level of intra-group competition (Figure 3C). Population size is determined by population growth thus changes in population growth rate may have significant impact on population size. A reduction in the population growth of PGYP1 individuals (Figure 4B) suggests that, relative to uninfected wild type populations, when Wolbachia infection is maintained at high frequency in a field population, relatively fewer mosquitoes may emerge per developmental site during mosquito outbreaks (e.g., during wet seasons) because of increased competition for resources during the larval stage. In addition to the reduced survival time of wMelPop infected embryos and adults in A. aegypti (McMeniman et al., 2009; McMeniman and O'Neill, 2010; Yeap et al., 2011), successful establishment of the wMelPop infection in the field may act to suppress population size of $A$. aegypti, further contributing reduction of disease transmission by Wolbachia infected population.

Although CI level, relative fecundity of infected females, and maternal inheritance of Wolbachia infection are major parameters that determine the infection dynamics (Hoffmann et al., 1990), they are relatively difficult to modify in terms of efficiently promoting Wolbachia invasion in the release studies. Thus, when infection cost increases the unstable equilibrium point that needs to be exceeded to initiate Wolbachia invasion (Turelli, 2010), available options are limited to increasing the initial infection frequency since the spread of Wolbachia is infection frequency dependent. The initial infection frequency can be increased either by increasing the release number of infected individuals or decreasing the population size in target areas. However the former option should examine whether or not the increased release number of infected individuals elevates the competition level at immature stages and reduces the relative fitness of infected individuals. Alternatively, reducing the field population size may decrease the larval density in the field and subsequently reduce the risk of increased resource competition, such that infected larvae suffer fewer fitness costs from the Wolbachia infection, which consequently facilitates the invasion of Wolbachia into field populations. Consistent with this hypothesis, Wolbachia invasions into natural populations have been indeed easier on the locations occupied with lower number of uninfected individuals (Hoffmann et al., 2014a). Thus, as was proposed in previous model studies that assessed male biased release of infected individuals (Hancock et al., 2011) and insecticide application on wild type populations (Hoffmann and Turelli, 2013), strategies to reduce the size of field populations could be helpful for promoting the spread of infection that cause high fitness cost to the hosts (Hoffmann, 2014). While further studies should examine whether or not the level of larval competition is increased by removal of larval habitats, direct removal of larval populations might be more helpful for reducing the risk of increased larval competition as well as reducing the population size (Jacups et al., 2013).

The negative impact of $w$ MelPop infection on population growth might have been associated with difficulties in establishing the infection in the field (Nguyen et al., 2015), since only a $5 \%$ reduction in relative survival of infected larvae could make population replacement difficult in a model study (Crain et al., 2011). In contrast, wMel infected individuals were over-represented with larger size when directly competing with uninfected larvae, which was not observed for wMelPop infection in a prior study (Ross et al., 2014). Taken together, larval competitiveness of $A$. aegypti could be greater with wMel relative to wMelPop infection which also imposes diverse fitness cost in other life-history traits, suggesting $w \mathrm{Mel}$ infection could be more successful for population replacement strategy, which is consistent 
with prior studies (Hoffmann et al., 2014b; Hoffmann et al., 2011; Nguyen et al., 2015; Walker et al., 2011). Thus, the use of wMelPop infection in applied strategies might be more appropriate for areas with particular ecological and environmental conditions (e.g., little mosquito migrations, low population size, etc.) as suggested in a prior study (Rasic et al., 2014). Lastly, evaluating the effect of $w \mathrm{Mel}$ infection on the population growth of infected populations might be also useful to better understand the population dynamics and its impact on disease transmission after establishment of the infection in the field.

\section{Acknowledgments}

We thank S. O'Neill for providing mosquito strains (PGYP1 and PGYP1.tet). We thank Adam F. Lindstrom for statistical advice. We thank Dr. Dong-Hwan Choe for helpful comments on the paper. This research was supported by grants from the National Institutes of Health [AI-067434] and the Bill and Melinda Gates Foundation [\#44190]. The information reported in this paper (No. 17-08-009) is part of a project of the Kentucky Agricultural Experiment Station and is published with the approval of the Director.

\section{References}

Aliota MT, Peinado SA, Velez ID, Osorio JE. The wMel strain of Wolbachia reduces transmission of Zika virus by Aedes aegypti. Sci Rep. 2016

Bian G, Joshi D, Dong Y, Lu P, Zhou G, Pan X, Xu Y, Dimopoulos G, Xi Z. Wolbachia invades Anopheles stephensi populations and induces refractoriness to Plasmodium infection. Science. 2013; 340:748-751. [PubMed: 23661760]

Bian GW, Xu Y, Lu P, Xie Y, Xi ZY. The endosymbiotic bacterium Wolbachia induces resistance to dengue virus in Aedes aegypti. PLoS Pathog. 2010; 6:e1000833. [PubMed: 20368968]

Blagrove MSC, Arias-Goeta C, Failloux AB, Sinkins SP. Wolbachia strain wMel induces cytoplasmic incompatibility and blocks dengue transmission in Aedes albopictus. Proceedings of the National Academy of Sciences of the United States of America. 2012; 109:255-260. [PubMed: 22123944]

Bourtzis K, Dobson SL, Xi Z, Rasgon JL, Calvitti M, Moreira LA, Bossin HC, Moretti R, Baton LA, Hughes GL, Mavingui P, Gilles JR. Harnessing mosquito- Wolbachia symbiosis for vector and disease control. Acta Tropica. 2014; 132:150-163.

Braks MAH, Honorio NA, Lounibos LP, Lourenco-De-Oliveira R, Juliano SA. Interspecific competition between two invasive species of container mosquitoes, Aedes aegypti and Aedes albopictus (Diptera : Culicidae), in Brazil. Ann Entomol Soc Am. 2004; 97:130-139.

Chrostek E, Marialva MSP, Esteves SS, Weinert LA, Martinez J, Jiggins FM, Teixeira L. Wolbachia variants induce differential protection to viruses in Drosophila melanogaster a phenotypic and phylogenomic analysis. PloS Genetics. 2013; 9

Crain PR, Mains JW, Suh E, Huang YX, Crowley PH, Dobson SL. Wolbachia infections that reduce immature insect survival: predicted impacts on population replacement. BMC Evolutionary Biology. $2011 ; 11$

Dutra HL, Rocha MN, Dias FB, Mansur SB, Caragata EP, Moreira LA. Wolbachia blocks currently circulating Zika virus isolates in Brazilian Aedes aegypti mosquitoes. Cell Host Microbe. 2016; 19:771-774. [PubMed: 27156023]

Ferguson NM, Kien DTH, Clapham H, Aguas R, Trung VT, Chau TNB, Popovici J, Ryan PA, O'Neill SL, McGraw EA, Long VT, Dui LT, Nguyen HL, Chau NVV, Wills B, Simmons CP. Modeling the impact on virus transmission of Wolbachia-mediated blocking of dengue virus infection of Aedes aegypti. Science Translational Medicine. 2015; 7

Frentiu FD, Zakir T, Walker T, Popovici J, Pyke AT, van den Hurk A, McGraw EA, O’Neill SL. Limited dengue virus replication in field-collected Aedes aegypti mosquitoes infected with Wolbachia. Plos Neglect Trop Dis. 2014; 8

Gavotte L, Mercer DR, Stoeckle JJ, Dobson SL. Costs and benefits of Wolbachia infection in immature Aedes albopictus depend upon sex and competition level. J Invertebr Pathol. 2010; 105:341-346. [PubMed: 20807539] 
Gavotte L, Mercer DR, Vandyke R, Mains JW, Dobson SL. Wolbachia infection and resource competition effects on immature Aedes albopictus (Diptera: Culicidae). J Med Entomol. 2009; 46:451-459. [PubMed: 19496412]

Hancock PA, Sinkins SP, Godfray HCJ. Strategies for introducing Wolbachia to reduce transmission of mosquito-borne diseases. PLoS Negl Trop Dis. 2011; 5

Hancock PA, White VL, Ritchie SA, Hoffmann AA, Godfray HC. Predicting Wolbachia invasion dynamics in Aedes aegypti populations using models of density-dependent demographic traits. BMC Biol. 2016; 14

Hedges LM, Brownlie JC, O’Neill SL, Johnson KN. Wolbachia and virus protection in Insects. Science. 2008; 322:702-702. [PubMed: 18974344]

Hilgenboecker K, Hammerstein P, Schlattmann P, Telschow A, Werren JH. How many species are infected with Wolbachia? - a statistical analysis of current data. FEMS Microbiol Lett. 2008; 281:215-220. [PubMed: 18312577]

Hoffmann AA. Facilitating Wolbachia invasions. Austral Entomol. 2014; 53:125-132.

Hoffmann AA, Goundar AA, Long SA, Johnson PH, Ritchie SA. Invasion of Wolbachia at the residential block level is associated with local abundance of Stegomyia aegypti, yellow fever mosquito, populations and property attributes. Med Vet Entomol. 2014a; 28:90-97. [PubMed: 25171611]

Hoffmann AA, Iturbe-Ormaetxe I, Callahan AG, Phillips B, Billington K, Axford JK, Montgomery B, Turley AP, O'Neill SL. Stability of the wMel Wolbachia infection following invasion into Aedes aegypti populations. Plos Neglect Trop Dis. 2014b; 8

Hoffmann AA, Montgomery BL, Popovici J, Iturbe-Ormaetxe I, Johnson PH, Muzzi F, Greenfield M, Durkan M, Leong YS, Dong Y, Cook H, Axford J, Callahan AG, Kenny N, Omodei C, McGraw EA, Ryan PA, Ritchie SA, Turelli M, O'Neill SL. Successful establishment of Wolbachia in Aedes populations to suppress dengue transmission. Nature. 2011; 476:454-U107. [PubMed: 21866160]

Hoffmann AA, Turelli M. Facilitating Wolbachia introductions into mosquito populations through insecticide-resistance selection. Proc R Soc B-Biol Sci. 2013; 280

Hoffmann AA, Turelli M, Harshman LG. Factors affecting the distribution of cytoplasmic incompatibility in Drosophila simulans. Genetics. 1990; 126:933-948. [PubMed: 2076821]

Iturbe-Ormaetxe I, Walker T, Neill SLO. Wolbachia and the biological control of mosquito-borne disease. EMBO Rep. 2011; 12:508-518. [PubMed: 21546911]

Jacups SP, Ball TS, Paton CJ, Johnson PH, Ritchie SA. Operational Use of Household Bleach to "Crash and Release" Aedes aegypti Prior to Wolbachia-Infected Mosquito Release. Journal of Medical Entomology. 2013; 50:344-351. [PubMed: 23540123]

Jeong G, Suh E. Wolbachia-induced reproductive anomalies and their future applications. Entomological Research. 2008; 38:41-48.

Juliano SA. Species introduction and replacement among mosquitoes: interspecific resource competition or apparent competition? Ecology. 1998; 79:255-268.

Kambris Z, Blagborough AM, Pinto SB, Blagrove MSC, Godfray HCJ, Sinden RE, Sinkins SP. Wolbachia stimulates immune gene expression and inhibits Plasmodium development in Anopheles gambiae. PLoS Pathog. 2010; 6:e1001143. [PubMed: 20949079]

Kambris Z, Cook PE, Phuc HK, Sinkins SP. Immune activation by life-shortening Wolbachia and reduced filarial competence in mosquitoes. Science. 2009; 326:134-136. [PubMed: 19797660]

Kho EA, Hugo LE, Lu G, Smith DD, Kay BH. Effects of larval nutrition on Wolbachia-based dengue virus interference in Aedes aegypti (Diptera: Culicidae). J Med Entomol. 2016; 53:894-901. [PubMed: 27106932]

Koenraadt CJM, Kormaksson M, Harrington LC. Effects of inbreeding and genetic modification on Aedes aegypti larval competition and adult energy reserves. Parasit Vectors. 2010; 3

Livdahl TP, Sugihara G. Non-linear interactions of populations and the importance of estimating percapita rates of change. J Anim Ecol. 1984; 53:573-580.

Livdahl TP, Willey MS. Prospects for an invasion: competition between Aedes albopictus and native Aedes triseriatus. Science. 1991; 253:189-191. [PubMed: 1853204]

McGraw EA, O’Neill SL. Beyond insecticides: new thinking on an ancient problem. Nat Rev Microbiol. 2013; 11:181-193. [PubMed: 23411863] 
McMeniman CJ, Hughes GL, O’Neill SL. A Wolbachia symbiont in Aedes aegypti disrupts mosquito egg development to a greater extent when mosquitoes feed on nonhuman versus human blood. J Med Entomol. 2011; 48:76-84. [PubMed: 21337952]

McMeniman CJ, Lane AM, Fong AWC, Voronin DA, Iturbe-Ormaetxe I, Yamada R, McGraw EA, O'Neill SL. Host adaptation of a Wolbachia atrain after long-term serial passage in mosquito cell lines. Appl Environ Microbiol. 2008; 74:6963-6969. [PubMed: 18836024]

McMeniman CJ, Lane RV, Cass BN, Fong AWC, Sidhu M, Wang YF, O’Neill SL. Stable introduction of a life-shortening Wolbachia infection into the mosquito Aedes aegypti. Science. 2009; 323:141144. [PubMed: 19119237]

McMeniman CJ, O'Neill SL. A virulent Wolbachia infection decreases the viability of the dengue vector Aedes aegypti during periods of embryonic quiescence. PLoS Negl Trop Dis. 2010; 4:e748. [PubMed: 20644622]

Min KT, Benzer S. Wolbachia, normally a symbiont of Drosophila, can be virulent, causing degeneration and early death. Proc Natl Acad Sci USA. 1997; 94:10792-10796. [PubMed: 9380712]

Moreira LA, Iturbe-Ormaetxe I, Jeffery JA, Lu GJ, Pyke AT, Hedges LM, Rocha BC, Hall-Mendelin S, Day A, Riegler M, Hugo LE, Johnson KN, Kay BH, McGraw EA, van den Hurk AF, Ryan PA, O’Neill SL. A Wolbachia symbiont in Aedes aegypti limits infection with dengue, Chikungunya, and Plasmodium. Cell. 2009; 139:1268-1278. [PubMed: 20064373]

Nguyen TH, Le Nguyen H, Nguyen TY, Vu SN, Tran ND, Le TN, Vien QM, Bui TC, Le HT, Kutcher S, Hurst TP, Duong TTH, Jeffery JAL, Darbro JM, Kay BH, Iturbe-Ormaetxe I, Popovici J, Montgomery BL, Turley AP, Zigterman F, Cook H, Cook PE, Johnson PH, Ryan PA, Paton CJ, Ritchie SA, Simmons CP, O’Neill SL, Hoffmann AA. Field evaluation of the establishment

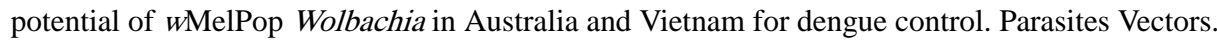
$2015 ; 8$

Rasic G, Endersby NM, Williams C, Hoffmann AA. Using Wolbachia-based release for suppression of Aedes mosquitoes: insights from genetic data and population simulations. Ecol Appl. 2014; 24:1226-1234. [PubMed: 25154109]

Ritchie S, Townsend M, Paton C, Callahan A, Hoffmann A. Application of wMelPop Wolbachia strain to crash local populations of Aedes aegypti. Plos Neglect Trop Dis. 2015; 9(7):e0003930.

Ross PA, Endersby NM, Hoffmann AA. Costs of three Wolbachia infections on the survival of Aedes aegypti larvae under starvation conditions. Plos Neglect Trop Dis. 2016; 10

Ross PA, Endersby NM, Yeap HL, Hoffmann AA. Larval competition extends developmental time and decreases adult size of wMelPop Wolbachia-infected Aedes aegypti. Am J Trop Med Hyg. 2014; 91:198-205. [PubMed: 24732463]

Suh E, Dobson SL. Reduced competitiveness of Wolbachia infected Aedes aegypti larvae in intra- and inter-specific immature interactions. J Invertebr Pathol. 2013; 114:173-177. [PubMed: 23933013]

Suh E, Mercer DR, Dobson SL. Interaction of Wolbachia and bloodmeal type in artificially infected Aedes albopictus (Diptera: Culicidae). Journal of Medical Entomology. 2016:tjw084. [PubMed: 27313167]

Suh E, Mercer DR, Fu YQ, Dobson SL. Pathogenicity of life-shortening Wolbachia in Aedes albopictus after transfer from Drosophila melanogaster. Appl Environ Microbiol. 2009; 75:77837788. [PubMed: 19820149]

Turelli M. Cytoplasmic incompatibility in populations with overlapping generations. Evolution. 2010; 64:232-241. [PubMed: 19686264]

Turley AP, Moreira LA, O’Neill SL, McGraw EA. Wolbachia infection reduces blood-feeding success in the dengue fever mosquito, Aedes aegypti. Plos Neglect Trop Dis. 2009; 3:e516.

Van den Hurk AF, Hall-Mendelin S, Pyke AT, Frentiu FD, McElroy K, Day A, Higgs S, O’Neill SL. Impact of Wolbachia on infection with chikungunya and yellow fever viruses in the mosquito vector Aedes aegypti. Plos Neglect Trop Dis. 2012; 6

Walker T, Johnson PH, Moreira LA, Iturbe-Ormaetxe I, Frentiu FD, McMeniman CJ, Leong YS, Dong Y, Axford J, Kriesner P, Lloyd AL, Ritchie SA, O’Neill SL, Hoffmann AA. The wMel Wolbachia strain blocks dengue and invades caged Aedes aegypti populations. Nature. 2011; 476:450-U101. [PubMed: 21866159] 
Werren JH, Baldo L, Clark ME. Wolbachia: master manipulators of invertebrate biology. Nat Rev Microbiol. 2008; 6:741-751. [PubMed: 18794912]

Ye YH, Carrasco AM, Frentiu FD, Chenoweth SF, Beebe NW, Hurk AFvd, Simmons CP, O'Neill SL, McGraw EA. Wolbachia reduces the transmission potential of dengue-infected Aedes aegypti. Plos Neglect Trop Dis. 2015

Ye YXH, Woolfit M, Rances E, O’Neill SL, McGraw EA. Wolbachia-associated bacterial protection in the mosquito Aedes aegypti. Plos Neglect Trop Dis. 2013; 7

Yeap HL, Axford JK, Popovici J, Endersby NM, Iturbe-Ormaetxe I, Ritchie SA, Hoffmann AA. Assessing quality of life-shortening Wolbachia-infected Aedes aegypti mosquitoes in the field based on capture rates and morphometric assessments. Parasites Vectors. 2014; 7

Yeap HL, Mee P, Walker T, Weeks AR, O’Neill SL, Johnson P, Ritchie SA, Richardson KM, Doig C, Endersby NM, Hoffmann AA. Dynamics of the "popcorn" Wolbachia infection in outbred Aedes aegypti informs prospects for mosquito vector control. Genetics. 2011; 187:583-U346. [PubMed: 21135075]

Zug R, Hammerstein P. Still a host of hosts for Wolbachia: Analysis of recent data suggests that $40 \%$ of terrestrial arthropod species are infected. PLoS One. 2012; 7 


\section{Highlights}

- Effect of Wolbachia on immature mosquitoes is larval density dependent

- Wolbachia did not affect population growth of mosquitoes at low larval densities

- Wolbachia reduced population growth of mosquitoes at high larval densities 

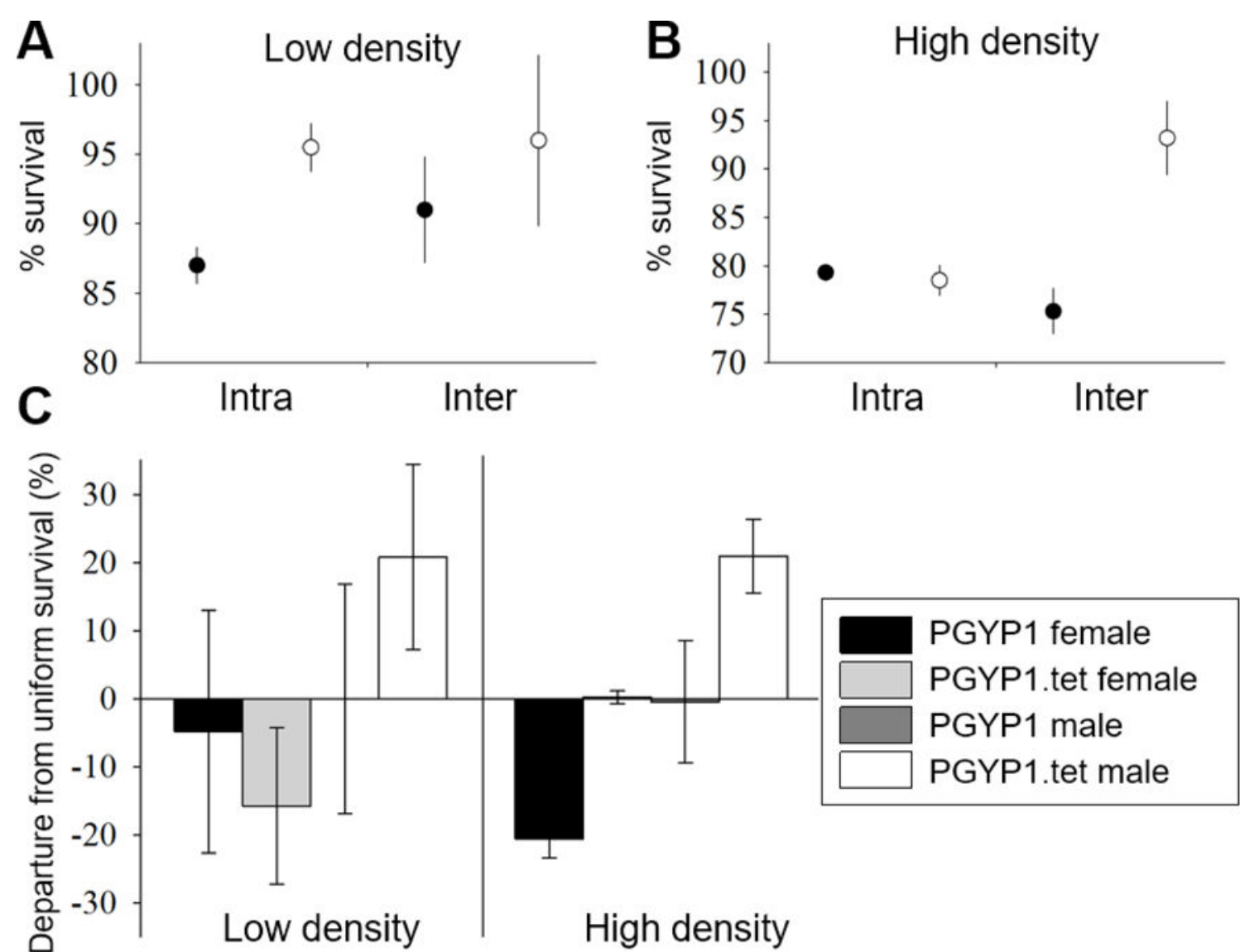

High density

Figure 1.

Survival of PGYP1 ( $w$ MelPop infected $A$. aegypti; solid circle) and PGYP1.tet (uninfected $A$. aegypt; open circle) in intra- and inter-group competition at low (A) and high (B) larval density. Departure from an equal emergence of $A$. aegypti competing at two larval density conditions $(\mathrm{C})$. Error bars = s.e.m. 

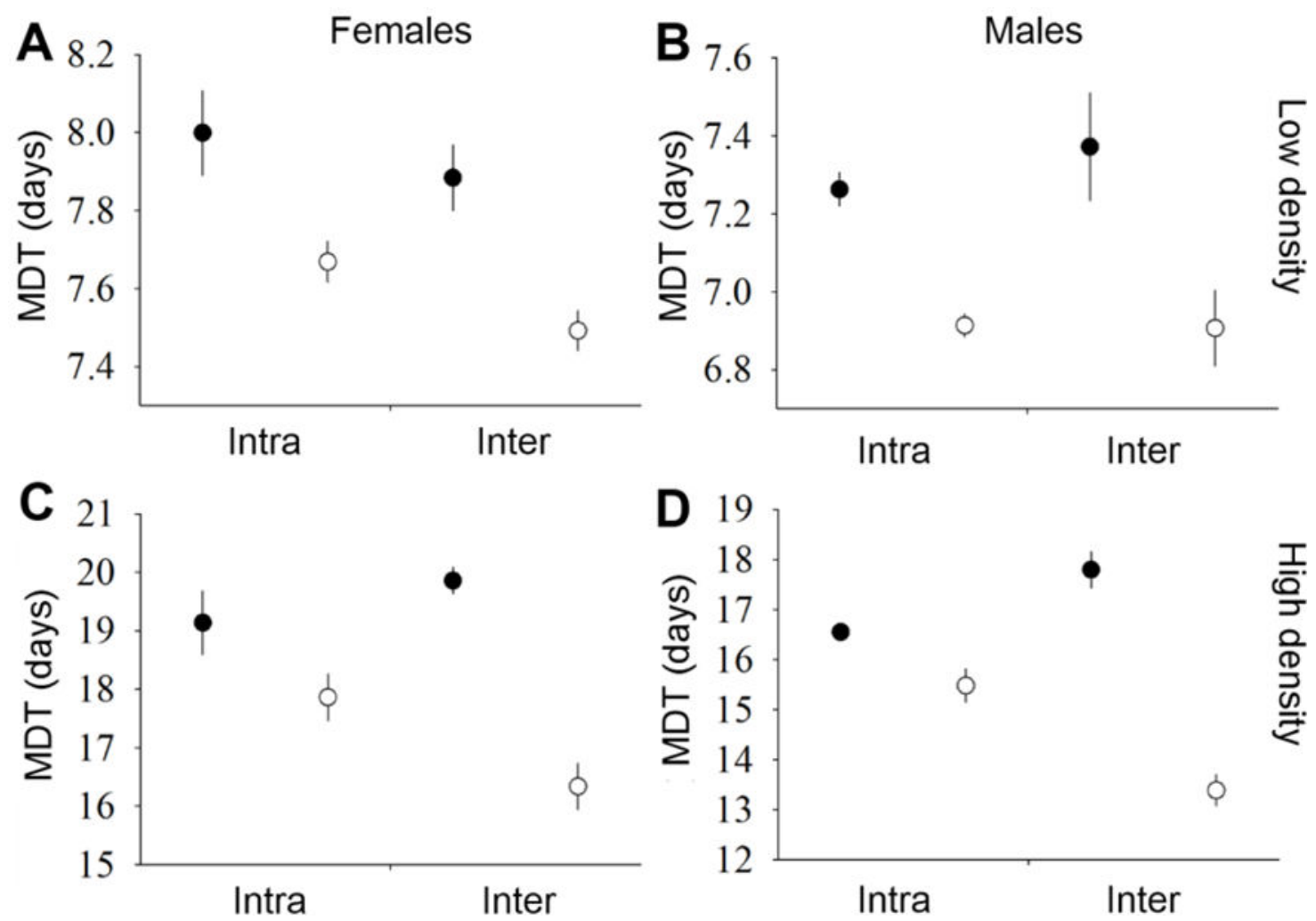

Figure 2.

Mean development time (MDT) of PGYP1 ( $w$ MelPop infected $A$. aegypti; solid circle) and PGYP1.tet (uninfected $A$. aegypt; open circle) females (A, C) and males (B, D) in intra- and inter-group competition at low $(\mathrm{A}, \mathrm{B})$ and high $(\mathrm{C}, \mathrm{D})$ larval density conditions. Error bars = s.e.m. 

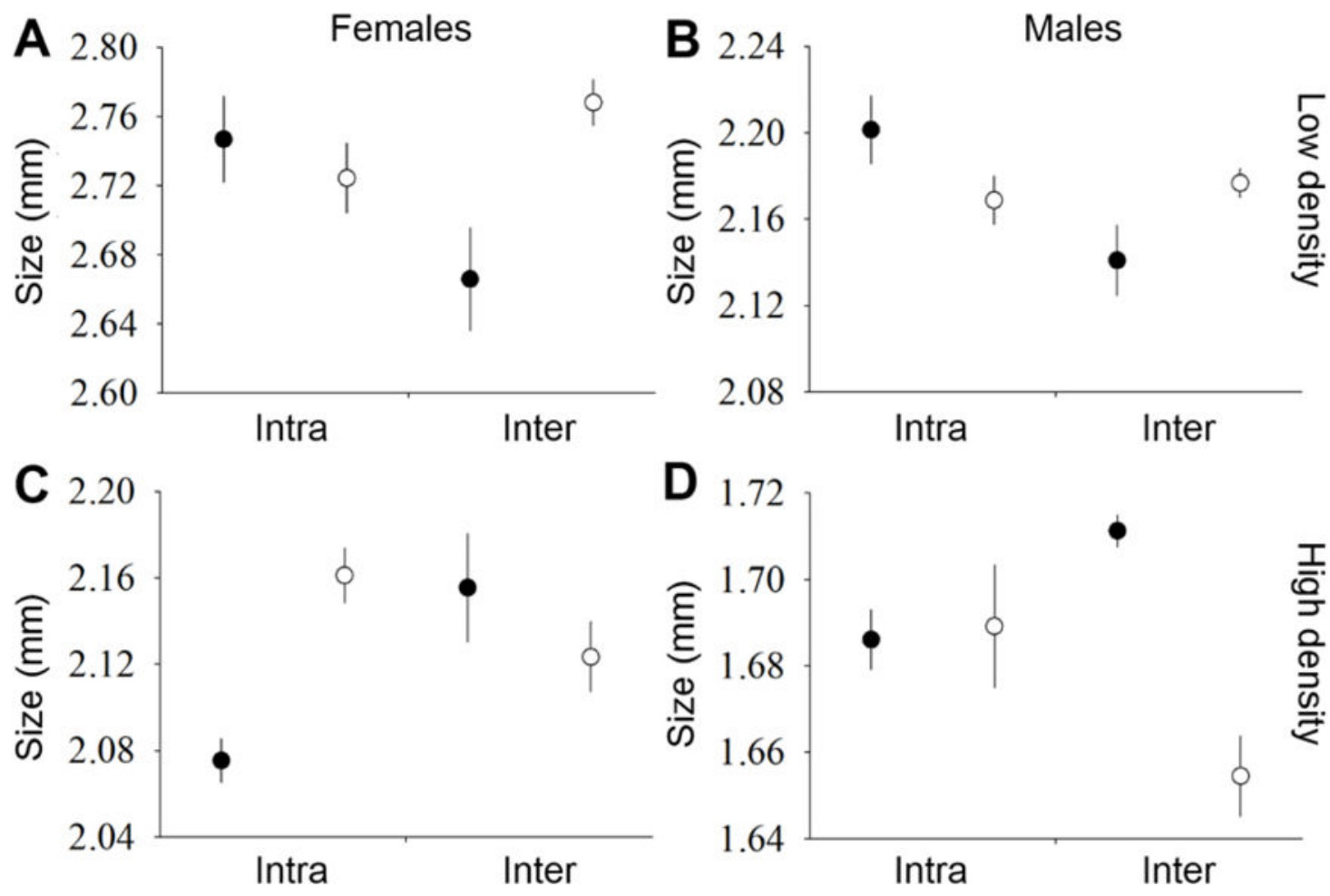

Figure 3.

Wing size of PGYP1 ( $w$ MelPop infected A. aegypt; solid circle) and PGYP1.tet (uninfected $A$. aegypti; open circle) females (A, C) and males (B, D) in intra- and inter-group competition at low $(\mathrm{A}, \mathrm{B})$ and high $(\mathrm{C}, \mathrm{D})$ larval density conditions. Error bars = s.e.m. 

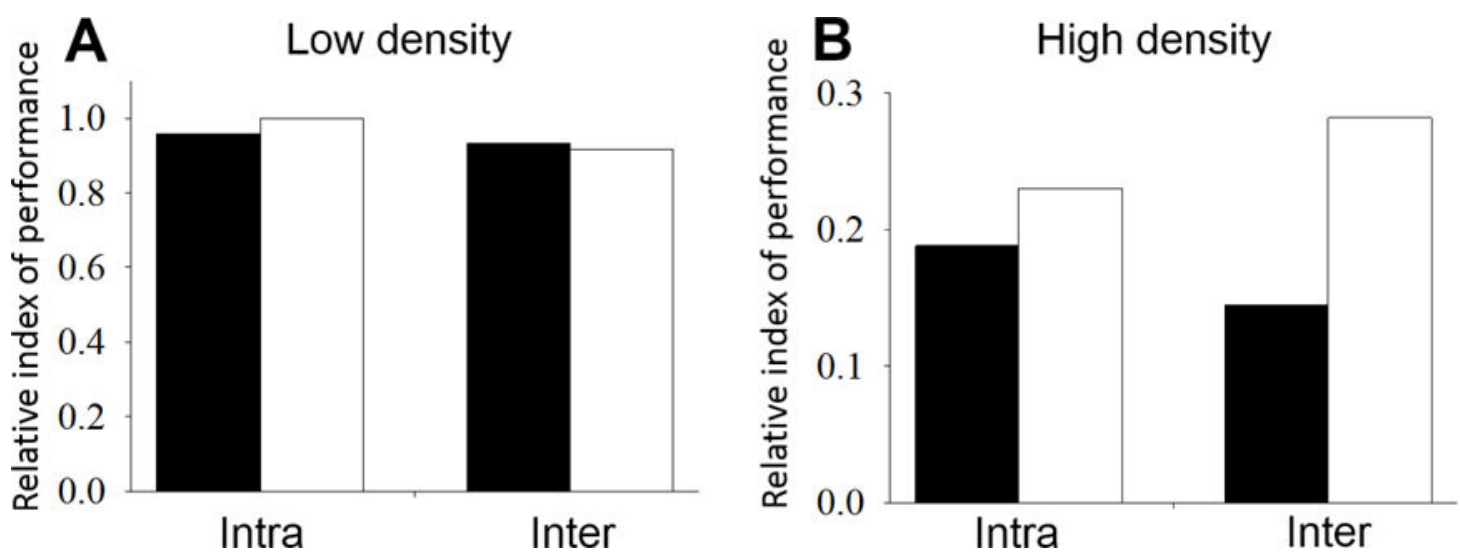

Figure 4.

Relative performance index of PGYP1 ( $w$ MelPop infected $A$. aegypti; solid) and PGYP1.tet (uninfected $A$. aegypti; white) at low (A) and high (B) larval density. The relative performance index is the estimated rate of population growth. The standardized relative index was defined as 1 for PGYP1.tet individuals in intra-group competition under low density condition, and the performance index of the other groups was calculated based on the standardized relative index. 


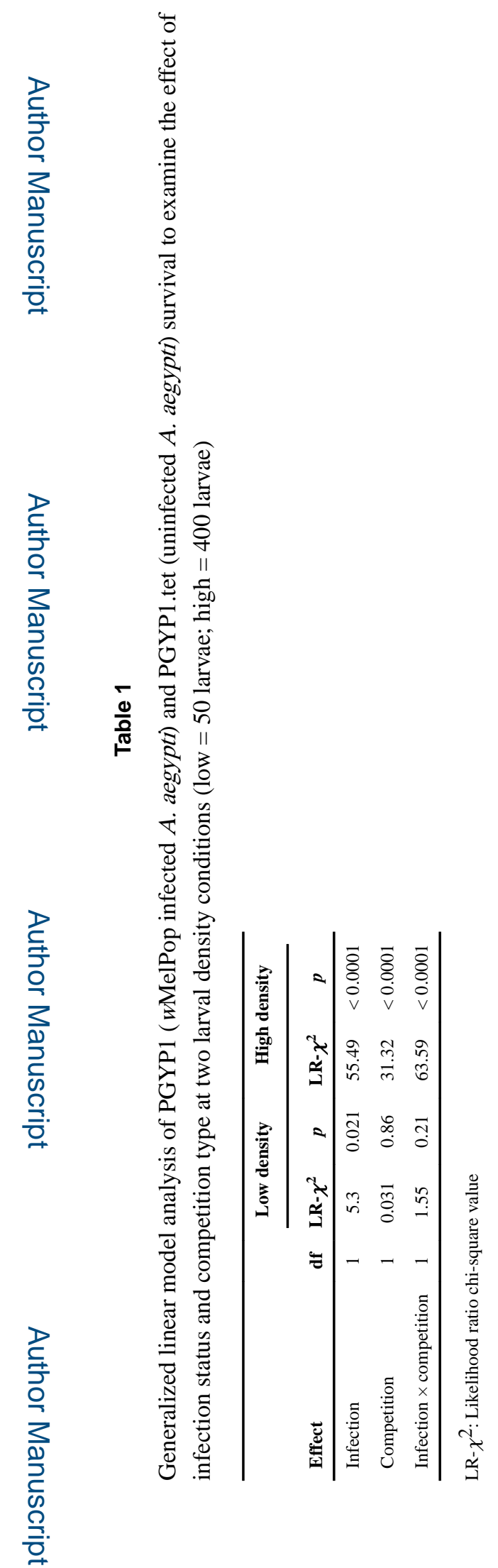

Acta Trop. Author manuscript; available in PMC 2018 August 01. 


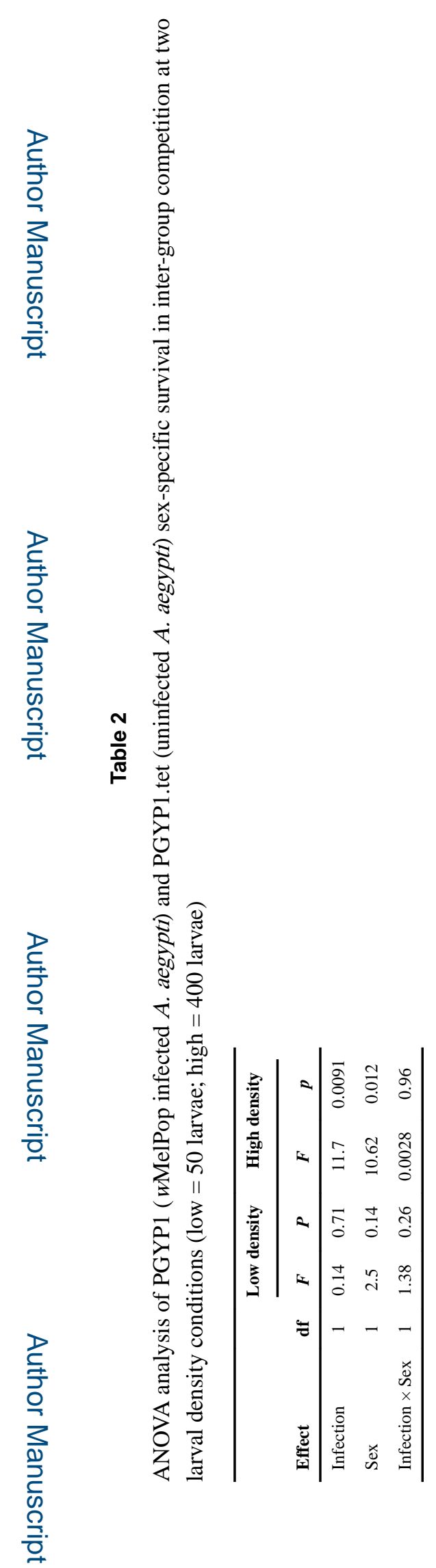

Acta Trop. Author manuscript; available in PMC 2018 August 01. 


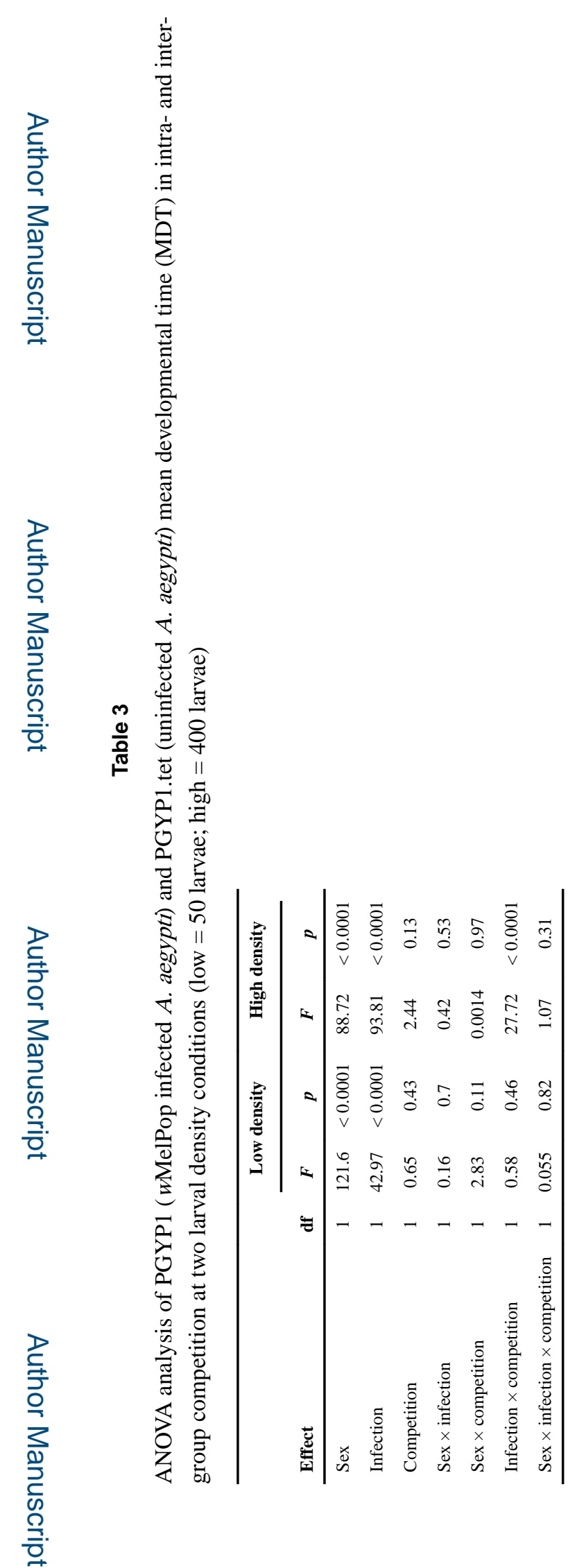

Acta Trop. Author manuscript; available in PMC 2018 August 01. 


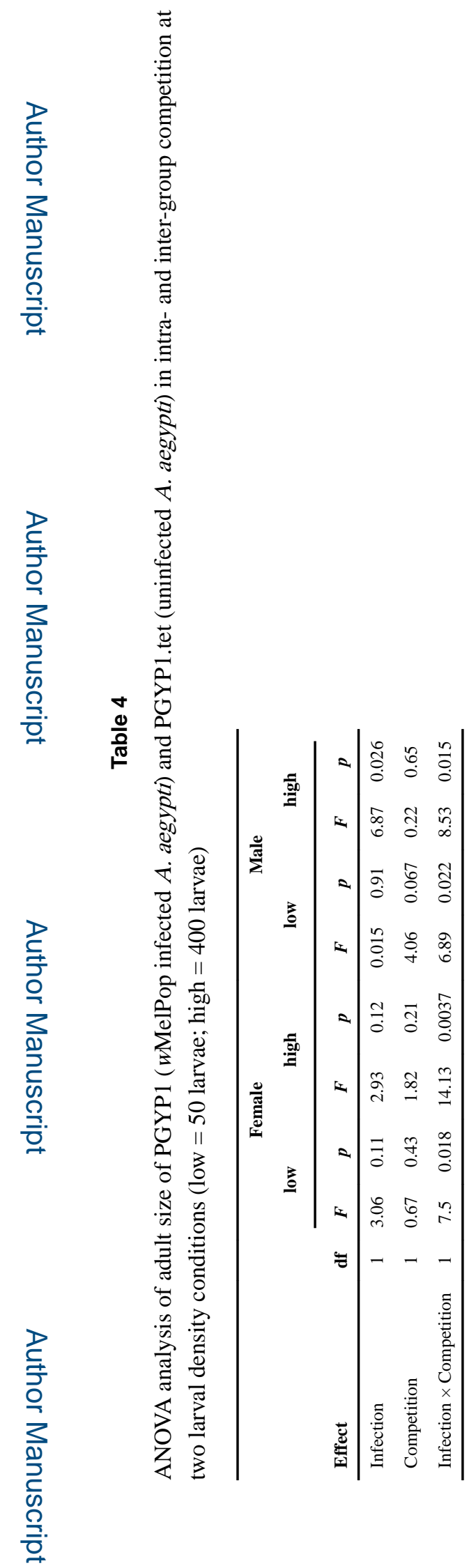

Acta Trop. Author manuscript; available in PMC 2018 August 01. 


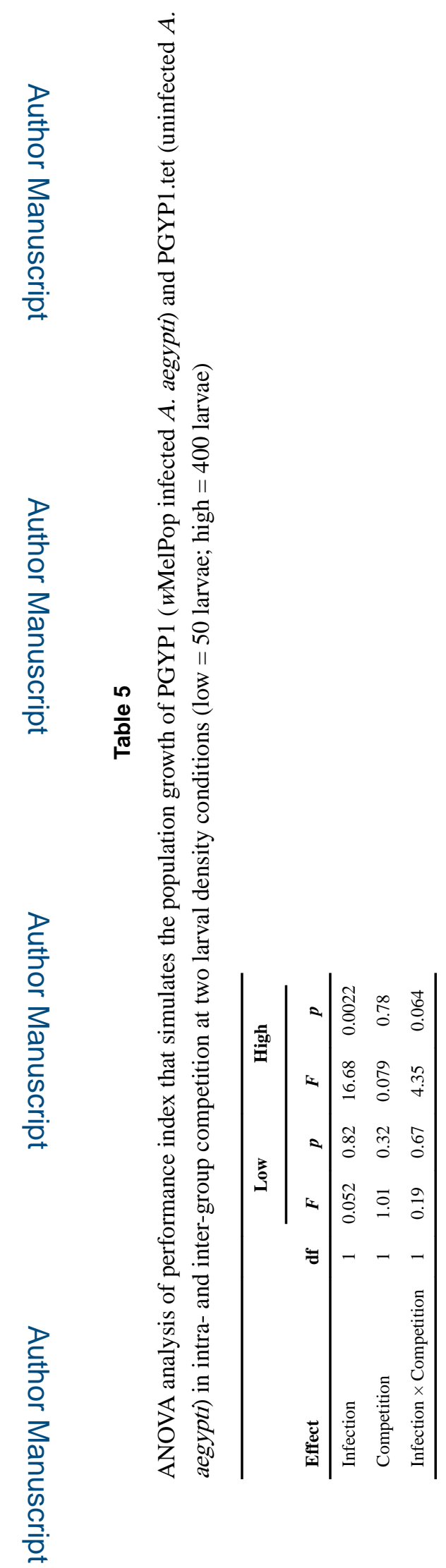

Acta Trop. Author manuscript; available in PMC 2018 August 01. 